\title{
Relation between occupation in the first coordination shells and Widom line in core- softened potentials
}

Cite as: J. Chem. Phys. 138, 164502 (2013); https://doi.org/10.1063/1.4802006

Submitted: 27 December 2012 . Accepted: 03 April 2013. Published Online: 23 April 2013

Evy Salcedo, Ney M. Barraz, and Marcia C. Barbosa

\section{ARTICLES YOU MAY BE INTERESTED IN}

Widom line and the liquid-liquid critical point for the TIP4P/2005 water model

The Journal of Chemical Physics 133, 234502 (2010); https://doi.org/10.1063/1.3506860

A general purpose model for the condensed phases of water: TIP4P/2005

The Journal of Chemical Physics 123, 234505 (2005); https://doi.org/10.1063/1.2121687

Structural properties and fragile to strong transition in confined water

The Journal of Chemical Physics 146, 084505 (2017); https://doi.org/10.1063/1.4975624

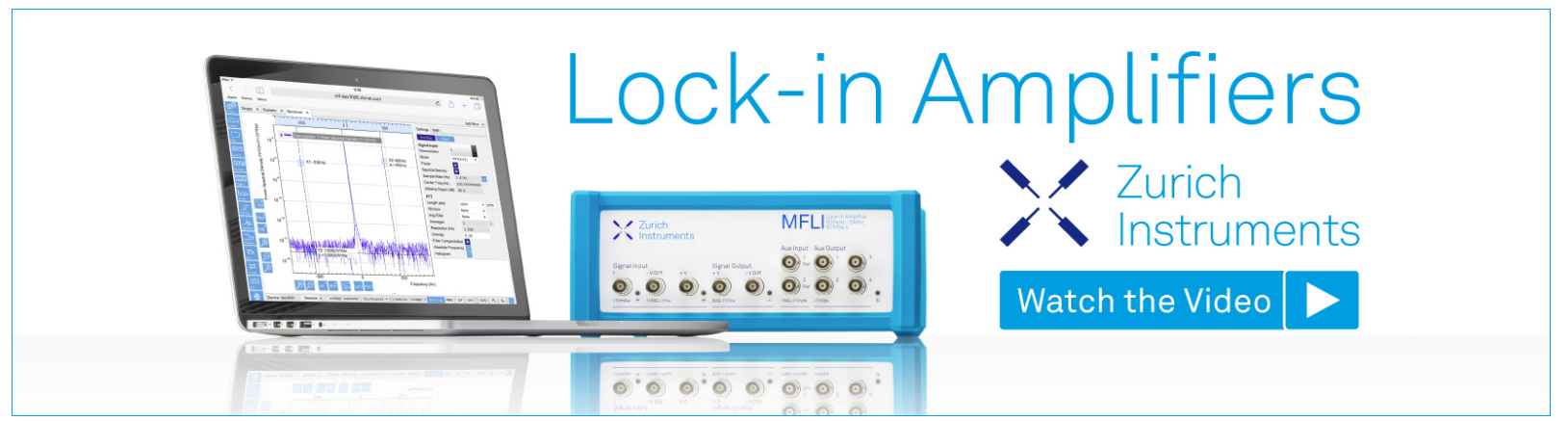

J. Chem. Phys. 138, 164502 (2013); https://doi.org/10.1063/1.4802006 


\title{
Relation between occupation in the first coordination shells and Widom line in core-softened potentials
}

\author{
Evy Salcedo, ${ }^{1}$ Ney M. Barraz, Jr., ${ }^{2}$ and Marcia C. Barbosa ${ }^{3}$ \\ ${ }^{1}$ Departamento de Física, Universidade Federal de Santa Catarina, 88010-970 Florianópolis, SC, Brazil \\ ${ }^{2}$ Universidade Federal da Fronteira Sul, 85303-160 Laranjeiras do Sul, Paraná, PR, Brazil \\ ${ }^{3}$ Instituto de Física, Universidade Federal do Rio Grande do Sul, 91501-970 Porto Alegre, \\ Rio Grande do Sul, Brazil
}

(Received 27 December 2012; accepted 3 April 2013; published online 23 April 2013)

\begin{abstract}
Three core-softened families of potentials are checked for the presence of density and diffusion anomalies. These potentials exhibit a repulsive core with a softening region and at larger distances an attractive well. We found that the region in the pressure-temperature phase diagram in which the anomalies are present increases if the slope between the core-softened scale and the attractive part of the potential decreases. The anomalous region also increases if the range of the core-softened or of the attractive part of the potential decreases. We also show that the presence of the density anomaly is consistent with the non-monotonic changes of the radial distribution function at each one of the two scales when temperature and density are varied. Then, using this anomalous behavior of the structure we show that the pressure and the temperature at which the radial distribution function of one of the two length scales equals the radial distribution function of the other length scales identify the Widom line. () 2013 AIP Publishing LLC. [http://dx.doi.org/10.1063/1.4802006]
\end{abstract}

\section{INTRODUCTION}

Core-softened $(C S)$ potentials have been attracting attention due to their connections with the anomalous behavior of liquid systems including water. These potentials, $\mathrm{U}(\mathrm{r})$, exhibit a repulsive core with a softening region limited by $r_{1}<r<r_{2}$, where $d(r f) / d r>0$ with $f=-d U / d r .{ }^{1}$ Despite their simplicity, these models originate from the desire of constructing a simple two-body isotropic potential capable of describing the complicated features of systems interacting via anisotropic potentials. ${ }^{2-27}$ This procedure generates models that are analytically tractable and computationally less expensive than the atomistic models. Moreover, they are lead to conclusions that are more universal and are related to families of atomistic systems. ${ }^{28-31}$

One of the features that has been successfully described by many of these models is the density anomaly. For water the specific volume at ambient pressure starts to increase when cooled below $T \approx 4{ }^{\circ} \mathrm{C}$. The anomalous behavior of water was first suggested $300 \mathrm{yr} \mathrm{ago}^{32}$ and was confirmed by a number of experiments. ${ }^{28,29}$ Besides, between $0.1 \mathrm{MPa}$ and $190 \mathrm{MPa}$ water also exhibits an anomalous increase of compressibility ${ }^{33,34}$ and, at atmospheric pressure, an increase of isobaric heat capacity upon cooling. ${ }^{35,36}$ For the case of water the density anomaly is attributed to the presence of hydrogen bonds between neighbor molecules. As the temperature increases the bonds break and the density increases. However, other systems such as $\mathrm{Te},{ }^{37} \mathrm{Ga}, \mathrm{Bi},{ }^{38}$ $\mathrm{S},{ }^{39,40} \mathrm{Ge}_{15} \mathrm{Te}_{85},{ }^{30}$ silica, ${ }^{31,41-43}$ silicon, ${ }^{44}$ and $\mathrm{BeF}_{2}{ }^{31}$ show the same density anomaly without presenting hydrogen bonds which suggests that the mechanism for the presence of density anomaly might be more universal.

In compass with the presence of the density anomaly in water a few years ago it was suggested that there are two liquid phases, a low density liquid (LDL) and a high density liquid (HDL). ${ }^{45}$ The critical point ending this transition, found only in computer simulations, is located at the supercooled region beyond the line of homogeneous nucleation and thus cannot be experimentally measured. Even with this limitation, this hypothesis has been supported by indirect experimental results. ${ }^{18,33,46}$ The presence of two liquid phases and of a second critical point is also observed in certain $C S$ potentials. $^{5-18,22-27}$

Which are the conditions for a CS potential to exhibit density anomaly and two liquid phases? A definitive answer to this question is still missing. There are, however, a few clues. If a CS potential is discontinuous it presents two liquid phases but no density anomaly ${ }^{47}$ is observed. However, once the CS potential is modified to be continuous, the anomalies appear. ${ }^{23}$

Recently it has been proposed that a CS potential exhibits density anomaly if the two length scales identified with the softened region are accessible $22,24,48$ and what can be understood is as follows. The radial distribution, $g(r)$, of a CS potential has peaks at $g\left(r_{1}\right)$ and $g\left(r_{2}\right)$, where $r_{1}$ and $r_{2}>r_{1}$ are the two length scales of the CS potential. ${ }^{1,20}$ If $\partial g(r) /\left.\partial \rho\right|_{r=r_{1}} \partial g(r) /\left.\partial \rho\right|_{r=r_{2}}<0$ then the system would have density anomaly.

In this paper we test if this link between the behavior of the structure (radial distribution function) and the thermodynamic anomalies holds for a number of two length scales potentials. We study the pressure temperature phase diagram of a two Fermi model. ${ }^{49}$ The advantage of this model is that by changing few parameters it is possible to vary the distance and the difference in energy between the two length scales without introducing extra scales. Moreover the length scales are well defined.

Hence, having identified a connection between the density anomaly and the behavior of the structure, we test if the 
TABLE I. Parameters for potentials $S, A$, and $R$ in reduced units of $\varepsilon$ and $\sigma=r_{o 1} / 0.950$.

\begin{tabular}{lccccccccc}
\hline \hline Parameter & $S_{1}$ & $S_{2}$ & $S_{3}$ & $A_{1}$ & $A_{2}$ & $A_{3}$ & $R_{1}$ & $R_{2}$ & $R_{3}$ \\
\hline$\varepsilon_{1}^{*}$ & 1.000 & 1.000 & 1.000 & 1.000 & 1.000 & 1.000 & 1.000 & 1.000 & 1.000 \\
$\varepsilon_{2}^{*}$ & 3.000 & 3.000 & 3.000 & 3.000 & 3.000 & 3.000 & 3.000 & 3.000 & 3.000 \\
$-\varepsilon_{3}^{*}$ & 1.000 & 1.027 & 1.023 & 1.023 & 1.023 & 1.023 & 1.023 & 1.023 & 1.023 \\
$r_{o 1}^{*}$ & 0.950 & 0.950 & 0.950 & 0.950 & 0.950 & 0.950 & 0.950 & 1.050 & 1.150 \\
$r_{o 2}^{*}$ & 1.400 & 1.400 & 1.400 & 1.400 & 1.400 & 1.400 & 1.400 & 1.400 & 1.400 \\
$r_{o 3}^{*}$ & 1.950 & 1.950 & 1.950 & 1.755 & 1.8525 & 1.950 & 1.950 & 1.950 & 1.950 \\
$\sigma_{1,2,3}^{*}$ & 0.025 & 0.040 & 0.055 & 0.025 & 0.025 & 0.025 & 0.055 & 0.055 & 0.055 \\
$\alpha_{1}^{*}$ & 0.000 & 0.000 & 0.000 & 0.000 & 0.000 & 0.000 & 0.000 & 0.000 & 0.000 \\
$\alpha_{2,3}^{*}$ & 1.000 & 1.000 & 1.000 & 1.000 & 1.000 & 1.000 & 1.000 & 1.000 & 1.000 \\
\hline \hline
\end{tabular}

radial distribution function is also related to the presence of two liquids predicted for these CS potentials. We show that the pressures and temperatures in which the radial distribution function associated with one scale equals the radial distribution function of the other scales are linked with peaks in the constant pressure specific heat, namely the Widom line.

The remaining of this paper goes as follows. In Sec. II the model is introduced and the simulation details are presented. In Sec. III the pressure-temperature phase diagram is presented together with the behavior of the radial distribution function with density and temperature. Conclusions are presented in Sec. IV.

\section{THE MODEL}

Our system consists of $N$ identical particles interacting through a continuous pair potential obtained by the addition of three different Fermi-Dirac distributions, ${ }^{49}$

$$
U=\sum_{i=1}^{3} \frac{\varepsilon_{i}}{\exp \left(\frac{r-r_{o i}}{\sigma_{i}}\right)+\alpha_{i}} .
$$

The resulting expression describes a family of pair interaction potentials discriminated by different choices of the parameters $\left\{\varepsilon_{i}, r_{o i}, \sigma_{i}, \alpha_{i}\right\}$. Appropriate choices of the parameters allow us to obtain potentials that go from a smooth two length scales potential to a sharp, almost discontinuous, square potential. ${ }^{49,50}$

In Table I nine different sets of parameters are shown, organized in three families named $S, A$, and $R$. As shown in Figure 1, for each family a specific characteristic of pair inter- action potential is tuned. Then it is possible to test the effect of changing the two length scales in the pressure temperature phase diagram.

In the potentials $S$ the slope between the two length scales is varied. Then it is possible to check if the slope between the two length scales controls the location in the pressuretemperature phase diagram of the density anomalous region as suggested by Yan et al. ${ }^{51}$

In the case of the potentials $A$, the attractive length becomes broader. Consequently using this potential we test if increasing the range of the attraction leads to a decrease in the critical pressure as proposed by Skibinsky et al. ${ }^{8}$

In the case of the potentials $R$, the repulsive length scale becomes broader. Therefore this family of potentials is appropriated to observe if the enlargement of the repulsive length scale leads to a decrease in the liquid-liquid critical pressure and to an increase in the liquid-liquid critical temperature as suggested by Skibinsky et al. ${ }^{8}$ In addition to verifying the assumptions of Yan et al. ${ }^{51}$ and of Skibinsky et al. ${ }^{8}$ related to criticality, these three families of potentials provide a perfect scenario to check our hypothesis that the density anomaly region in the pressure-temperature phase diagram is delimited by properties of the radial distribution function at the two length scales.

The thermodynamic and dynamic behavior of the systems were obtained using $N V T$ molecular dynamics using Nose-Hoover heat-bath with coupling parameter $Q=2$. The system is characterized by 500 particles in a cubic box with periodic boundary conditions, interacting with the intermolecular potential described above.
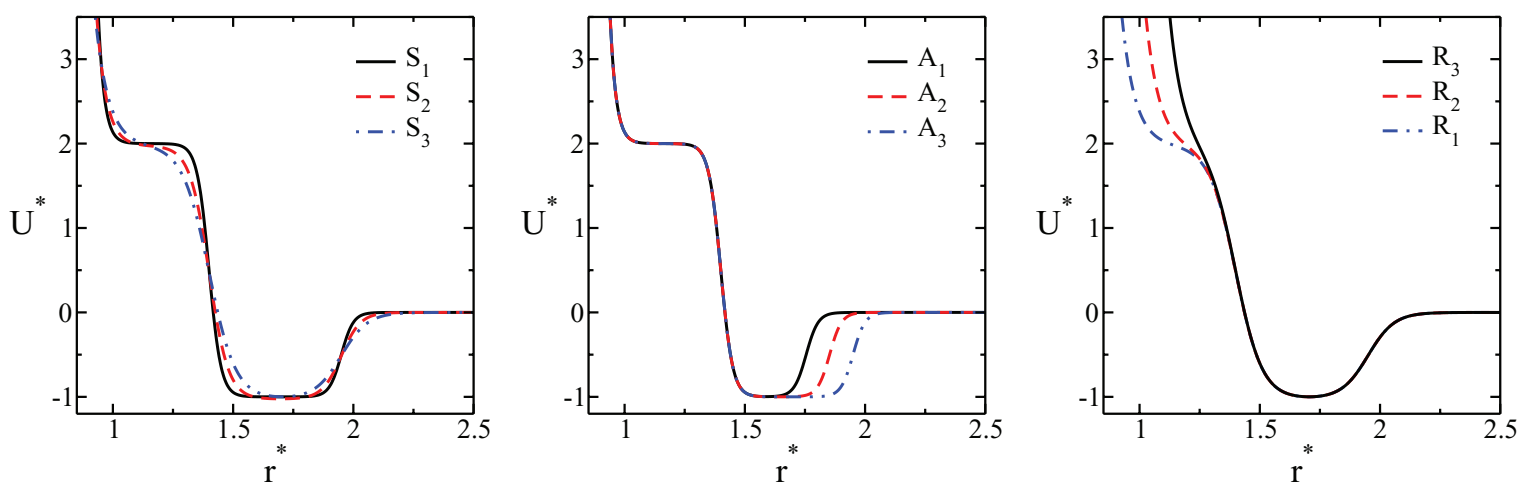

FIG. 1. Interaction potential. 

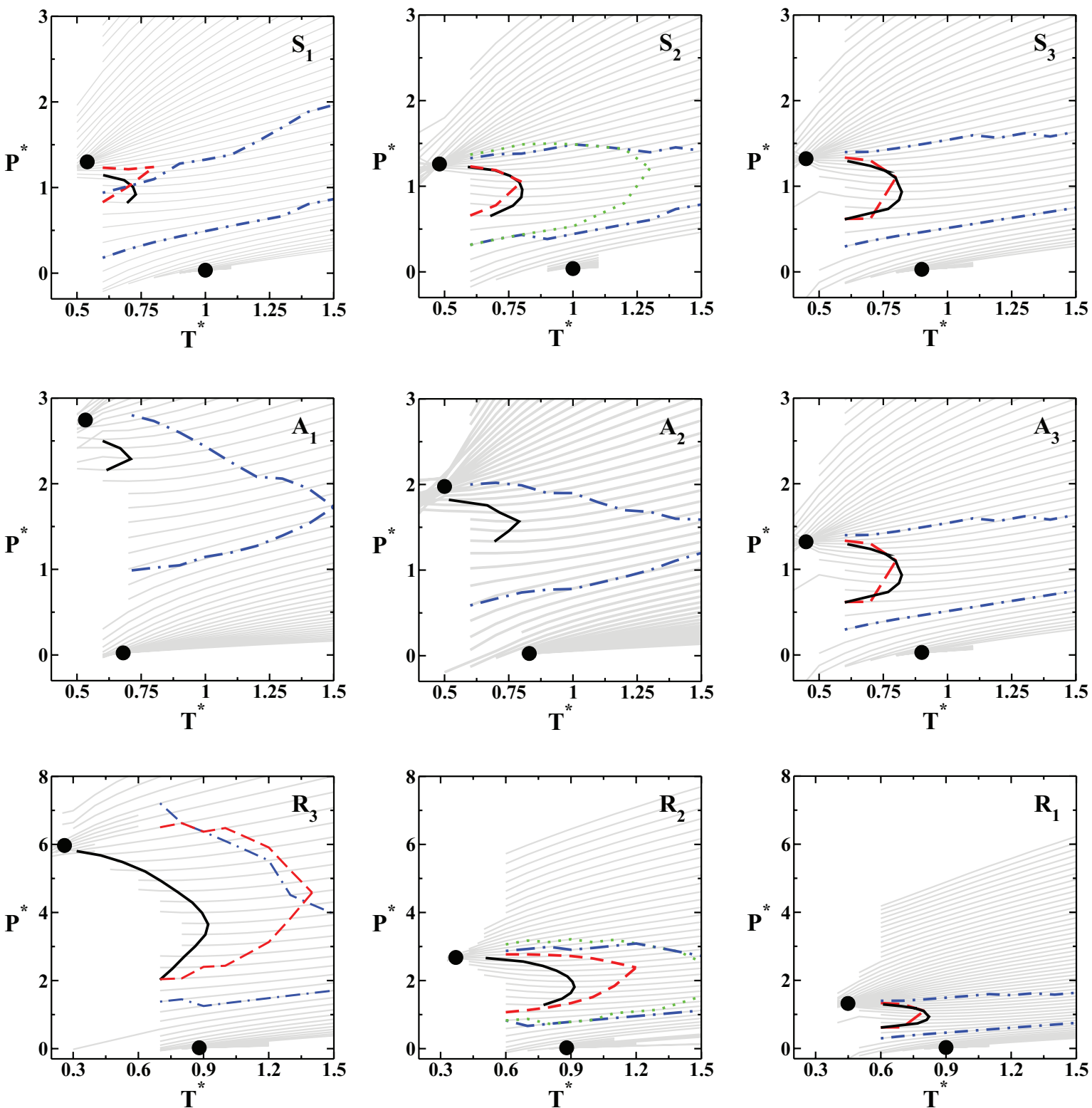

FIG. 2. Pressure-temperature phase space for the $S, A$, and $R$ families of potentials. The gray lines are isochores, the solid lines are the temperature of maximum density, the dashed lines are the extrema of diffusion, and the dotted-dashed line are the extrema of the translational order parameter. The filled circles are the liquid-gas (at high temperatures) and the liquid-liquid (low temperatures) critical points.

Standard periodic boundary conditions together with predictor-corrector algorithm were used to integrate the equations of motion with a time step $\Delta t^{*}=0.002$ and potential cut off radius $r_{c}^{*}=2.5$. The initial configuration is set on solid or liquid state and, in both cases, the equilibrium state was reached after $t_{e q}^{*}=1000$. From this time on the physical quantities were stored in intervals of $\Delta t_{R}^{*}=1$ during $t_{R}^{*}=1000$. The system is uncorrelated after $t_{d}^{*}=10$, from the velocity auto-correlation function, and 50 decorrelated samples were used to get the average of the physical quantities. The thermodynamic stability of the system was checked analyzing the dependence of pressure on density, by the behavior of the energy, and also by visual analysis of the final structure, searching for cavitation.

In what follows we take $\varepsilon_{1}$ and $\sigma=r_{o 1} / 0.950$ as fundamental units for energy and distance, respectively, and all physical quantities are expressed in reduced units, namely,

$$
\begin{aligned}
T^{*} & \equiv \frac{k_{B} T}{\varepsilon_{1}}, \\
\rho^{*} & \equiv \rho \sigma^{3}, \\
P^{*} & \equiv \frac{P \sigma^{3}}{\varepsilon_{1}}, \\
D^{*} & \equiv \frac{D\left(m / \varepsilon_{1}\right)^{1 / 2}}{\sigma},
\end{aligned}
$$

where $\mathrm{T}, \mathrm{P}$, and $\mathrm{D}$ are, respectively, temperature, pressure, and diffusion coefficient. The diffusion coefficient is obtained from the expression:

$$
D=\lim _{t \rightarrow \infty} \frac{\left\langle\left[\vec{r}_{j}\left(t_{0}+t\right)-\vec{r}_{j}\left(t_{0}\right)\right]^{2}\right\rangle_{t_{0}}}{6 t}
$$


TABLE II. Liquid-gas critical point location for potentials $S, A$, and $R$.

\begin{tabular}{lcccccccc}
\hline \hline Potential & $T_{c_{1}}^{*}$ & $p_{c_{1}}^{*}$ & Potential & $T_{c_{1}}^{*}$ & $p_{c_{1}}^{*}$ & Potential & $T_{c_{1}}^{*}$ & $p_{c_{1}}^{*}$ \\
\hline$S_{1}$ & 0.05 & 1.00 & $A_{1}$ & 0.04 & 0.68 & $R_{1}$ & 0.02 & 0.90 \\
$S_{2}$ & 0.05 & 0.99 & $A_{2}$ & 0.03 & 0.82 & $R_{2}$ & 0.04 & 0.88 \\
$S_{3}$ & 0.04 & 0.88 & $A_{3}$ & 0.05 & 0.90 & $R_{3}$ & 0.04 & 0.88 \\
\hline
\end{tabular}

where $\vec{r}_{j}(t)$ are the coordinates of particle $j$ at time $t$ and $\langle\cdots\rangle_{t_{0}}$ denotes an average over all particles and over all $t_{0}$.

The errors associated with pressure and temperature are $\Delta p^{*} \approx 0.005$ and $\Delta T^{*} \approx 0.01$.

\section{RESULTS}

\section{A. Pressure-temperature phase space}

Figure 2 presents the pressure versus temperature phase space obtained for the three families: $S, A$, and $R$. In all the nine cases the system exhibits at high temperatures a fluid phase, at intermediate temperatures and very low pressures a gas phase, and at intermediate pressures a low density liquid phase (LDL), while at very high pressures a high density liquid phase (HDL). The coexistence line between the gas and the low density liquid phases (not shown) ends in a gas-LDL critical point illustrated as a filled circle in Fig. 2. The LDLHDL coexistence line (not shown) ends in a LDL-HDL critical point, also shown as a filled circle. The two critical points are located in the pressure and temperature phase space by the point in which the isochores meet. The critical pressures and the critical temperatures values are confirmed by analyzing the slope of the pressure versus density at constant temperature phase space. The inflection points of these curves identify the critical points. For the other state points the slope of the pressure versus density phase space is also used as a check of stability.

Tables II and III and Fig. 3 summarize the values of the first (liquid-gas) and second (liquid-liquid) critical points and their changes in the $p-T$ phase space for the three families studied.

Figure 2 shows that in the family of potentials $S$ the values of the pressure and temperature of the liquid-gas and the liquid-liquid critical points are not sensitive to the change of slope as predicted by Yan et al. ${ }^{51}$ The $A$ family, also illus-
TABLE III. Liquid-liquid critical point location for potentials $S, A$, and $R$.

\begin{tabular}{lcccccccc}
\hline \hline Potential & $T_{c_{2}}^{*}$ & $p_{c_{2}}^{*}$ & Potential & $T_{c_{2}}^{*}$ & $p_{c_{2}}^{*}$ & Potential & $T_{c_{2}}^{*}$ & $p_{c_{2}}^{*}$ \\
\hline$S_{1}$ & 1.31 & 0.54 & $A_{1}$ & 2.75 & 0.53 & $R_{1}$ & 1.34 & 0.44 \\
$S_{2}$ & 1.26 & 0.48 & $A_{2}$ & 1.98 & 0.50 & $R_{2}$ & 2.67 & 0.37 \\
$S_{3}$ & 1.34 & 0.44 & $A_{3}$ & 1.33 & 0.44 & $R_{3}$ & 6.01 & 0.26 \\
\hline \hline
\end{tabular}

trated in Fig. 2, indicates that the temperature of the liquidgas critical point increases with the increase of the range of the attractive scale, while the temperature and the pressure of the liquid-liquid critical point decrease. This result indicates that if the attractive scale increases, the high density liquid requires less pressure to be formed while the gas phases exist for higher temperatures as predicted by Skibinstky et al.$^{8,52} \mathrm{In}$ the case $R$, shown in Fig. 2 as well, the liquid-liquid critical pressure decreases with the increase of the range of the repulsive scale. This result indicates that as the repulsive scale becomes broader, it requires less pressure for the high density liquid to be formed while the repulsive scale has almost no effect in the low density liquid-gas coexistence line, as also predicted by Skibinstky et al. ${ }^{8,52}$ A summary of the liquid-gas and liquid-liquid critical pressures and temperatures is shown in Fig. 3.

\section{B. Density, diffusion, and translational anomalies}

Figure 2 shows the temperature of maximum density (TMD) for all the nine studied cases as solid thick lines. For all the potentials $S, A$, and $R$ the TMD lines are observed. The limits of the TMD in the pressure-temperature phase space are shown in Table IV, where $p_{l}$ represents the values of $\left(\rho^{*}, T^{*}, p^{*}\right)$ for the point of the lowest pressure in the TMD line, $p_{m}$ is the point with the highest temperature, and $p_{h}$ is the point with the highest pressure.

The top three graphs in Fig. 2 show that the effect of decreasing the slope between the two length scales in the pair interaction potential is to move the TMD to higher temperatures. This result explains why the TMD is not observed in the discontinuous square well (DSW) model. ${ }^{8}$ As the slope increases the TMD pressure and temperature approach the amorphous region and the system becomes unstable. For slopes higher than $S_{1}$ case no anomalous behavior is observed.
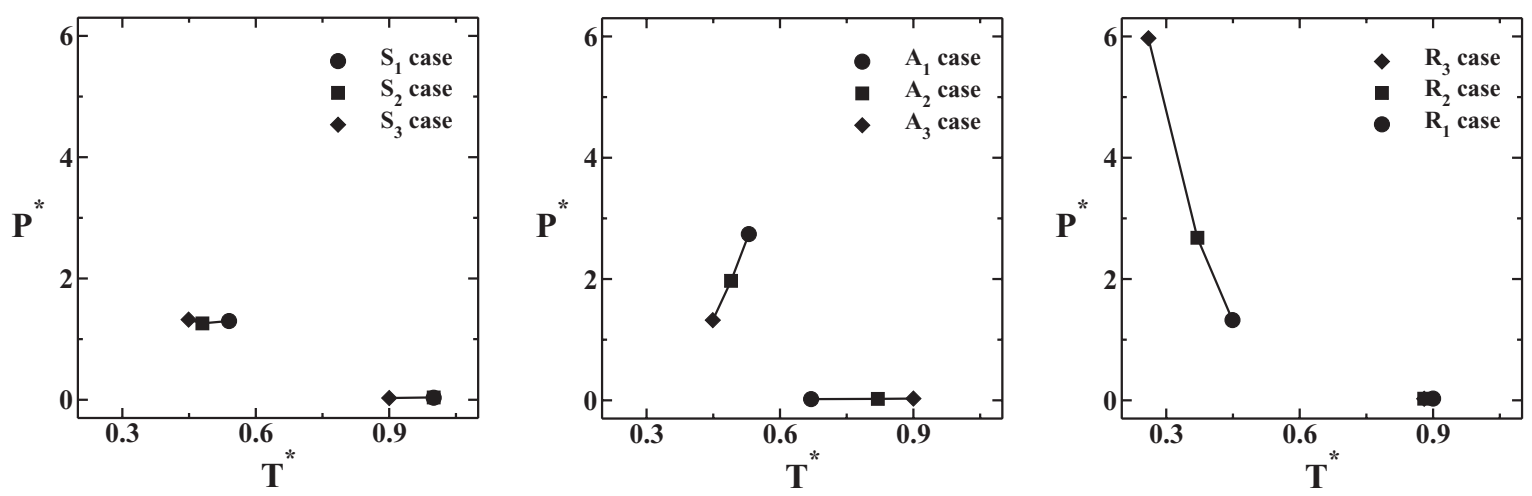

FIG. 3. Location of the critical points on pressure-temperature phase space for cases $S, A$, and $R$. 
TABLE IV. Limit values for density $\left(\rho^{*}\right)$, temperature $\left(T^{*}\right)$, and pressure $\left(p^{*}\right)$ of the thermodynamics anomalies on pressure-temperature space. Here the point $p_{l}$ represents the density, temperature, and pressure of the point of the lowest pressure in the TMD line, $p_{m}$ represents the point of the highest temperature, and $p_{h}$ represents the point of the highest pressure of the TMD line.

\begin{tabular}{ccccccccccccccc}
\hline \hline Case & & $p_{l}$ & $p_{m}$ & $p_{h}$ & Case & & $p_{l}$ & $p_{m}$ & $p_{h}$ & Case & & $p_{l}$ & $p_{m}$ & $p_{h}$ \\
\hline \multirow{4}{*}{$S_{1}$} & $\rho^{*}$ & 0.34 & 0.35 & 0.38 & & $\rho^{*}$ & 0.32 & 0.34 & 0.39 & & $\rho^{*}$ & 0.31 & 0.34 & 0.39 \\
& $T^{*}$ & 0.70 & 0.73 & 0.60 & $S_{2}$ & $T^{*}$ & 0.68 & 0.80 & 0.60 & $S_{3}$ & $T^{*}$ & 0.60 & 0.82 & 0.61 \\
& $p^{*}$ & 0.70 & 0.73 & 1.14 & & $p^{*}$ & 0.65 & 0.96 & 1.27 & & $p^{*}$ & 0.61 & 0.92 & 1.29 \\
& $\rho^{*}$ & 0.42 & 0.43 & 0.45 & & $\rho^{*}$ & 0.37 & 0.39 & 0.42 & & $\rho^{*}$ & 0.31 & 0.34 & 0.39 \\
$A_{1}$ & $T^{*}$ & 0.61 & 0.71 & 0.59 & \multirow{2}{*}{$A_{2}$} & $T^{*}$ & 0.51 & 0.79 & 0.69 & $A_{3}$ & $T^{*}$ & 0.60 & 0.82 & 0.61 \\
& $p^{*}$ & 2.15 & 2.29 & 2.51 & & $p^{*}$ & 1.33 & 1.56 & 1.82 & & $p^{*}$ & 0.61 & 0.92 & 1.29 \\
& $\rho^{*}$ & 0.31 & 0.34 & 0.39 & & $\rho^{*}$ & 0.33 & 0.37 & 0.42 & & $\rho^{*}$ & 0.34 & 0.39 & 0.47 \\
$R_{1}$ & $T^{*}$ & 0.60 & 0.82 & 0.61 & \multirow{2}{*}{$R_{2}$} & $T^{*}$ & 0.77 & 0.92 & 0.50 & $R_{3}$ & $T^{*}$ & 0.70 & 0.92 & 0.32 \\
& $p^{*}$ & 0.61 & 0.92 & 1.29 & & $p^{*}$ & 1.28 & 1.81 & 2.67 & & $p^{*}$ & 2.04 & 3.65 & 5.76 \\
\hline \hline
\end{tabular}
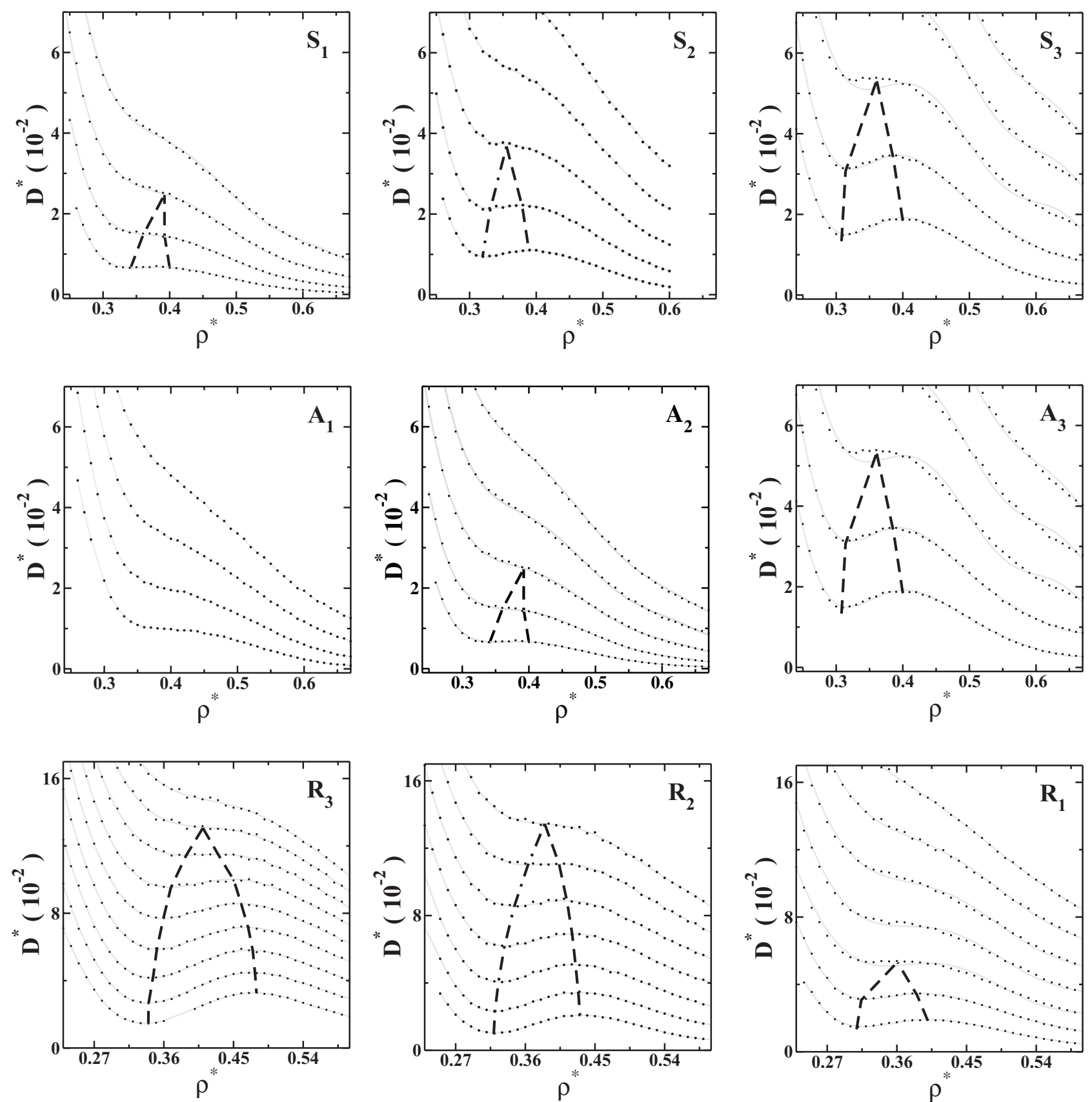

FIG. 4. Diffusion coefficient as a function of density. The dots are the simulation data and the solid lines are polynomial fits. The dashed lines connect the densities of minimal and maximal diffusivity that limit the diffusion anomalous region. 

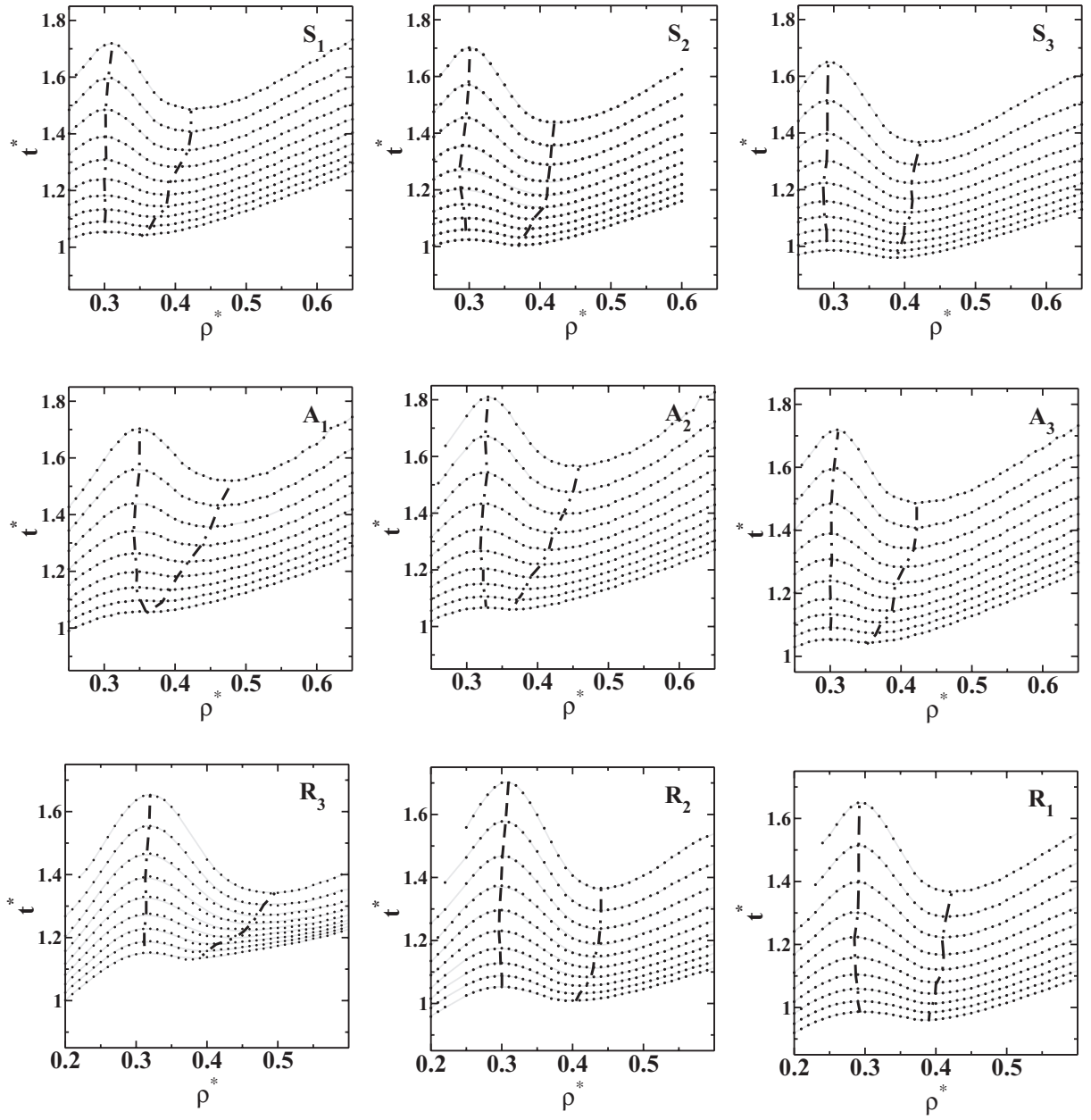

FIG. 5. The translational order parameter, a function of density for fixed temperatures $T^{*}=0.6,0.7,0.8,0.9,1.0,1.1,1.2,1.3,1.4,1.5$ (from top to bottom) for the families $S$ (on top), $A$ (on middle), and $R$ (on bottom). The dotted-dashed lines locate the maximal and minimal in $t^{*}$.

The middle graphs in Fig. 2 show that as the attractive scale increases, the TMD moves to higher temperatures and lower pressures as observed in potentials in which the attractive scale becomes dominant. ${ }^{53}$

The bottom graphs in Fig. 2 show that as the repulsive scale becomes broader, the density anomaly region in the pressure temperature phase space goes to lower pressures and shrinks as observed in potentials in which the repulsive scale becomes dominant. ${ }^{54}$

In addition, in all the phase spaces it is possible to observe that the TMD maximum pressure never exceeds the critical pressure. ${ }^{55}$ Our results indicate that the location in the pressure temperature phase space of the density anomalous region depends on the distance between the two length scales. ${ }^{52-54}$

Figure 4 shows the graphs of the dimensionless translational diffusion coefficient as a function of density for all families, $S, A$, and $R$. The solid gray lines are a polynomial fit to the data obtained by the simulations (the dots in Fig. 4). The diffusion coefficient follows the same trend of the TMD line. This result is not surprising since the hierarchy of the anomalies suggests that the mechanism for the presence of a TMD line is related to the mechanism for the existence of a maximum and minimum diffusion coefficient.
We also test the effects that changes in the two length scales have in the location in the pressure-temperature phase space of the structural anomalous region.

The translational order parameter is defined as ${ }^{42,56,57}$

$$
t=\int_{0}^{\xi_{c}}|g(\xi)-1| d \xi
$$

where $\xi=r \rho^{\frac{1}{3}}$ is the distance $r$ in units of the mean interparticle separation $\rho^{-\frac{1}{3}}, \xi_{c}$ is the cutoff distance set to half of the simulation box times ${ }^{20} \rho^{-\frac{1}{3}}$, and $g(\xi)$ is the radial distribution function which is proportional to the probability of finding a particle at a distance $\xi$ from a referent particle. The translational order parameter measures how structured the system is. For an ideal gas it is $g=1$ and $t=0$, while for the crystal phase it is $g \neq 1$ over long distances resulting in a large $t$. Therefore, for normal fluids $t$ increases with the increase of the density.

The graphs in Fig. 5 illustrate the translational order parameter versus density of the potentials studied. The dotteddashed lines show the maximum and minimum in the values of $t$ that limit the region of anomalous behavior. These extrema are also shown as dotted-dashed lines in Fig. 2. The values at the pressure-temperature phase space for the 

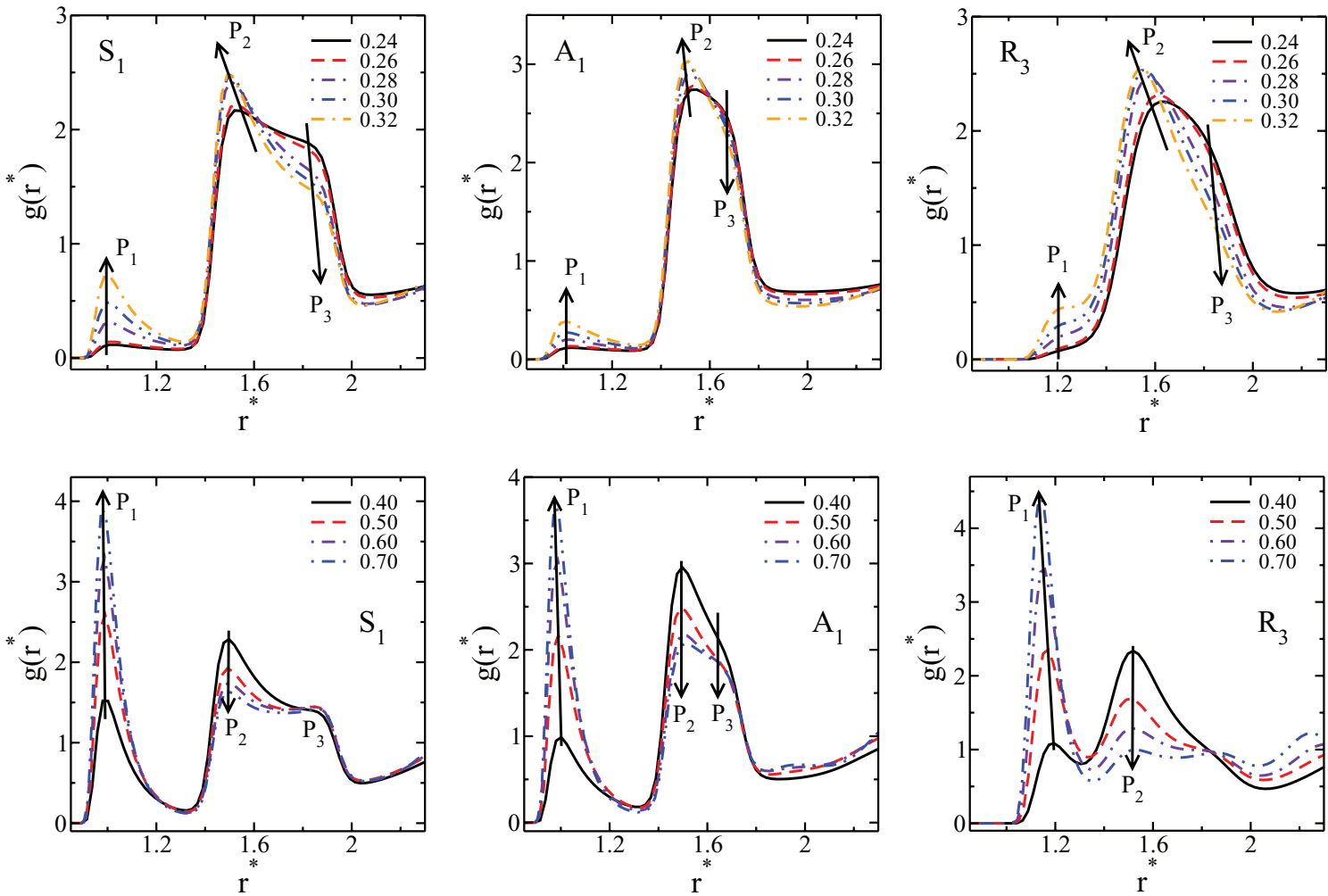

FIG. 6. Radial distribution as a function of the reduced distance for selected cases in the three families of potentials for $T^{*}=0.8$. For all the families, for $\rho^{*}<0.40$ the first and second peaks of $g(r)$ increase with the increase of density. For $\rho^{*} \geq 0.40$ the first peak increases while second peak decreases with the increase of density.

different potentials follow the same trend as the TMD and diffusion anomalous regions.

\section{Radial distribution function}

The density anomaly can be related to the structure by analyzing the behavior of the radial distribution function. For a two length scales potential the $g(r)$ has two peaks: one at the closest scale, $r_{1}$, and another at the furthest scale, $r_{2}{ }^{20}$

Recently it has been suggested that a signature of the presence of TMD line would be given by the radial distribution function as follows. At fixed temperature, as the density is increased the radial distribution function of the closest scale, $g\left(r_{1}\right)$, would increase its value while the radial distribution function of the furthest scale, $g\left(r_{2}\right)$, would decrease. This can be represented by the rule 22,48

$$
\Pi_{1,2}=\left.\frac{\partial g(r)}{\partial \rho}\right|_{r_{1}} \times\left.\frac{\partial g(r)}{\partial \rho}\right|_{r_{2}}<0
$$

The physical picture behind this condition ${ }^{22}$ is that for a fixed temperature as density increases particles that are located at the attractive scale, $r_{2}$, move to the repulsive scale, $r_{1}$. Figure 6 illustrates typical radial distribution functions for fixed $T^{*}$ as $\rho^{*}$ is varied. These graphs show that the picture of particles changing length scales due to pressure increase is valid for densities beyond a threshold density $\rho_{\min }^{*}$.
The regions identified by the radial distribution function as fulfilling the condition (5) are illustrated as opened circles in Fig. 7. The solid curve shows the TMD line. All the stable state points with density equal or higher than the minimum density at the TMD line verify the relation $\Pi_{1,2}(\rho, T)<0$. This result gives support to our assumption that the presence of anomalies is related to particles moving from the furthest scale, $r_{2}$, to closest length scale, $r_{1}$.

Figure 8 shows the value of the radial distribution function at the closest, $g\left(r_{1}\right)$ (dashed lines), and at the furthest scale, $g\left(r_{2}\right)$ (solid lines), as a function of the reduced density, $\rho^{*}$. For the closest scale $g\left(r_{1}\right)$ is monotonic with density while the value for $g\left(r_{2}\right)$ for a fixed temperature increases with the density for densities below $\rho^{*}<\rho_{\min }^{*}$ and decreases for densities above this threshold. This behavior, also shown in Fig. 6, corroborates the condition stated in Eq. (5) and supports the idea that particles move from one scale to the other by compression at $\rho>\rho_{\min }{ }^{22}$

Besides the move of particles from the attractive scale to the repulsive scale for $\rho^{*}>\rho_{\min }^{*}$ as the pressure (density) is increased, particles also move from one scale to the other due to the increase of temperature ${ }^{54}$ for $\rho^{*}<\rho_{\text {min }}^{*}$. At constant density, $\rho^{*}<\rho_{\min }^{*}$, the radial distribution function of the attractive scale, $g\left(r_{2}\right)$, decreases with the increase of the temperature while $g\left(r_{1}\right)$ increases with the increase of temperature, indicating that particles move from one scale to the other due to thermal effects. At the density $\rho_{\min }^{*}$, the value of $g\left(r_{1}\right)$ is independent of the temperature. 

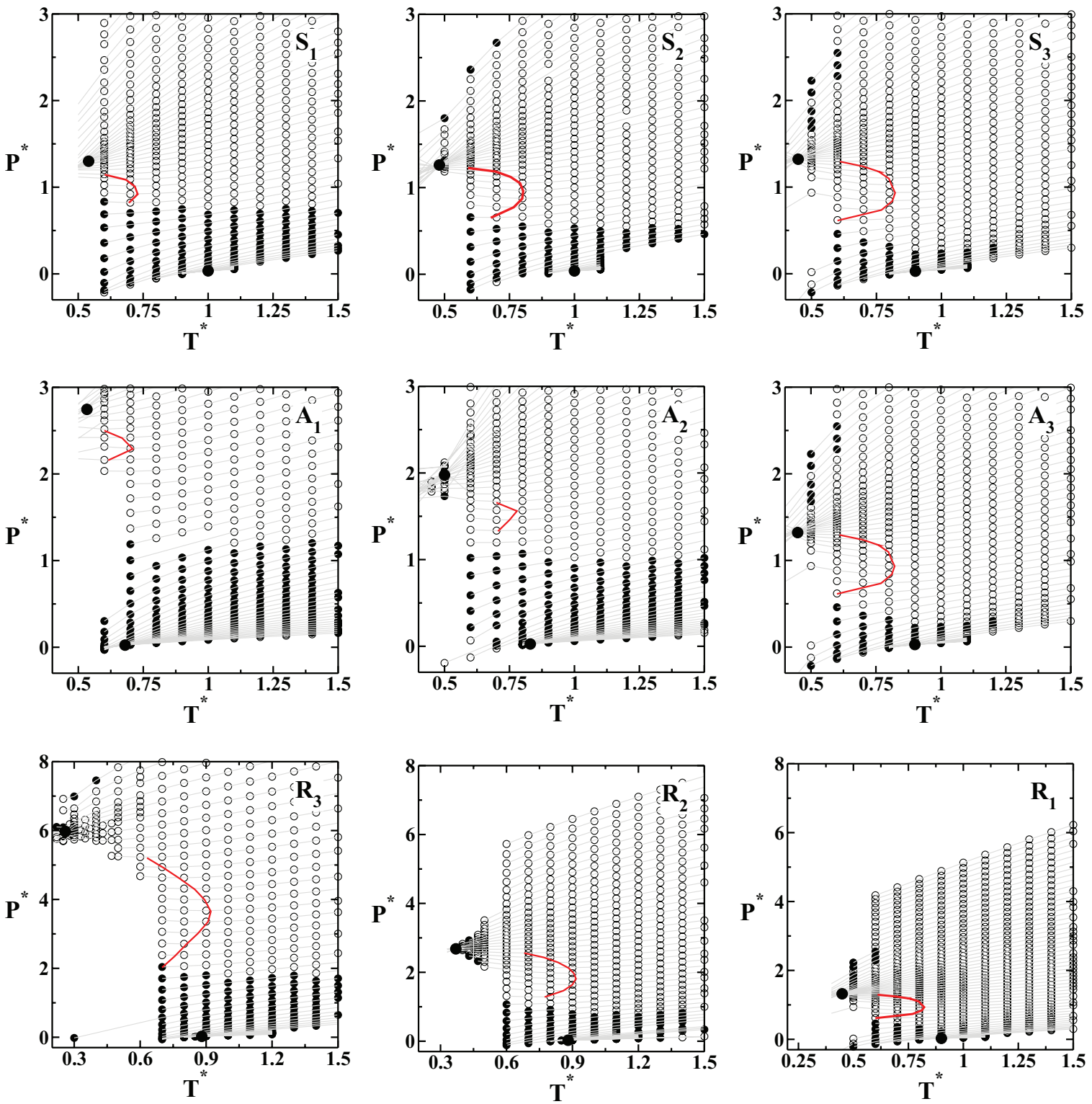

FIG. 7. Pressure-temperature phase space for the $S, A$, and $R$ families of potentials, illustrating as opened circles the regions where condition (5) is obeyed.

What is the meaning of the density $\rho_{\min }$ in which $g\left(r_{1}\right)$ is independent of temperature? As it was pointed in the previous paragraph, $\rho^{*}<\rho_{\min }^{*}$ particles move from the furthest scale, $r_{2}$, to the closest scale, $r_{1}$, using thermal energy as the temperature is increased. In this case $g\left(r_{1}\right)$ increases with temperature. $\rho^{*}>\rho_{\min }^{*}$ particles move from $r_{2}$ to $r_{1}$, using the increase in pressure (or density) as illustrated by Eq. (5). From a statistical point of view, the two mechanisms governing the behavior of $\rho^{*}>\rho_{\min }^{*}$ and $\rho^{*}<\rho_{\min }^{*}$ are quite different. While increasing the temperature affects particles individually, increasing the density or the pressure affects the particles as clusters or networks. Then, as the potential becomes more soft, the threshold density $\rho_{\min }^{*}$ beyond which the particles move from one scale to the other by compression should decrease as observed in Fig. 8. Therefore $\rho_{\min }^{*}$ is the threshold between these two mechanisms present in systems that have density anomaly.
In addition to these low density limit, the density anomalous systems also have a high density threshold, $\rho_{\max }^{*}(T)$. Figure 8 illustrates as a solid thick line the temperatures and densities, $\rho_{\max }^{*}(T)$, in which $g\left(r_{1}\right)=g\left(r_{2}\right)$. Since $g(r)$ is related with the number of particles at distance $r$, for $\rho^{*}<\rho_{\max }^{*}(T)$ more particles are in the attractive scale, $r_{2}$, while for $\rho^{*}>\rho_{\max }^{*}(T)$ more particles are at the repulsive scale, $r_{1}$. Therefore, the thick solid line is a boundary region in which the liquid starts to change from low density state to high density state or vice versa.

In order to understand what happens in the region of the pressure-temperature phase space of the $\rho_{\max }^{*}(T)$, the behavior of the specific heat in this region was analyzed. Figure 9 shows the curves of isobaric specific heat for different pressures as a function of the temperature for the potential $S_{1}$. The peak of $c_{p}$ for each one of the potentials analyzed in this manuscript coincides with the region $\rho_{\max }^{*}(T)$, where 

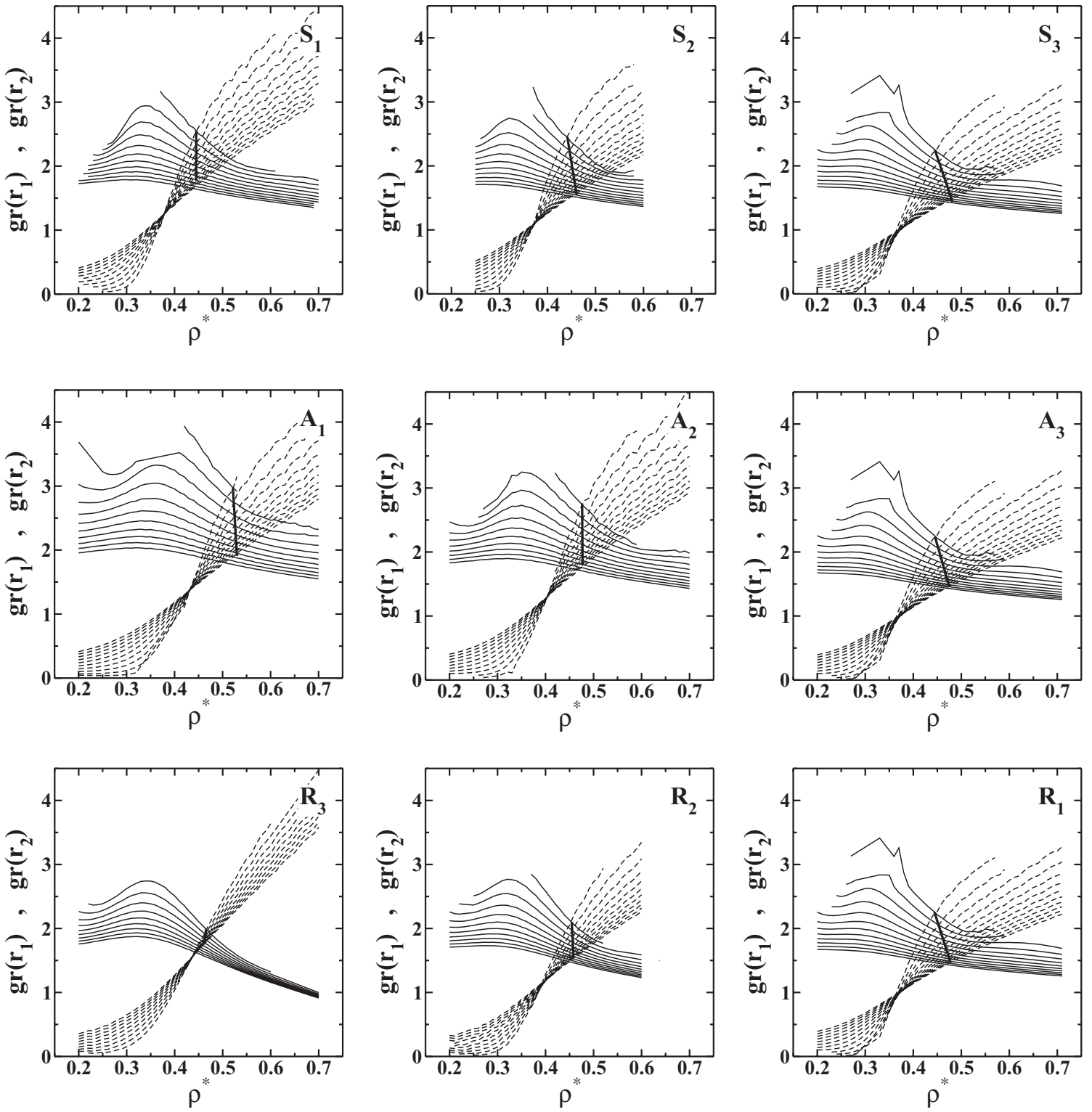

FIG. 8. $g\left(r_{1}\right)$ and $g\left(r_{2}\right)$ for the $S, A$, and $R$ families of potentials as a function of the reduced density. The temperatures are $T^{*}=0.4 \rightarrow 1.5$ (from top to bottom $\left.\rho^{*}>0.6\right)$. The solid line connects the points for different temperatures, where $g\left(r_{1}\right)=g\left(r_{2}\right)$.

$g\left(r_{1}\right)=g\left(r_{2}\right)$. This result indicates that the structure in the TMD region already builds the liquid arrangements required for the liquid-liquid phase separation.

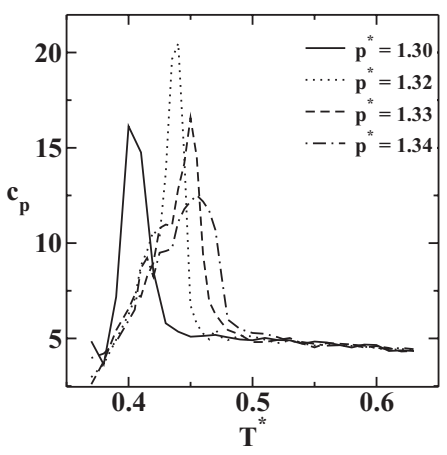

FIG. 9. Isobaric specific heat for the $S_{1}$ potential for different temperatures for different pressures.

\section{CONCLUSIONS}

In this paper we have studied three families of coresoftened potentials that exhibit two length scales, one repulsive, $r_{1}$, and another attractive, $r_{2}$.

We had observed that the region in the pressuretemperature phase space occupied by the TMD is quite sensitive to the slope between the two length scales, as the slope increases, the region decreases. We also found that the region in the pressure-temperature phase space, where the density, diffusion, and structural anomalous behavior is observed, shifts to lower pressures and shrinks as the attractive scales or the repulsive scales become wider. Our results suggest that the competition between two length scales are a relevant mechanism for the existence of the TMD.

In an attempt to confirm this assertion we showed in this family of potentials that the condition $\Pi_{1,2}<0$ seems to be associated with the presence of anomalous behavior. In 
addition we also observed that the peaks of the radial distribution function at each one of the two length scales exhibit very distinct behavior with density and temperature suggesting two complementary mechanisms for the competition between the two scales.

At low densities, $\rho^{*}<\rho_{\min }^{*}$ particles move from the scale at $r_{2}$ to the scale at $r_{1}$ with the increase of the temperature, using thermal energy. For densities above this threshold density, $\rho^{*}>\rho_{\min }^{*}$, the increase of radial distribution function at the length scale $r_{1}, g\left(r_{1}\right)$, is associated with the increase in the pressure (density). For our model the pressure and temperature in which the radial distribution function at one length scale equals the radial distribution at the other length scale, $g\left(r_{1}\right)=g\left(r_{2}\right)$, coincides with the location of the Widom line.

The relation between the radial distribution function and the Widom line in our model, believed to be the onset of the liquid-liquid phase transition, gives support to the idea that the Widom line separates two structurally distinct regions that are also separated by a fragile-strong transition. ${ }^{18}$

We expect that this result will not only shed some light in the definition of the shape an effective core-softened would have in order to hold anomalies but also would serve to reinforce the idea of linking dynamic transitions and thermodynamic properties.

\section{ACKNOWLEDGMENTS}

We thank for financial support the Brazilian science agencies $\mathrm{CNPq}$ and Capes. This work is partially supported by CNPq, INCT-FCx.

${ }^{1}$ P. G. Debenedetti, V. S. Raghavan, and S. S. Borick, J. Phys. Chem. 95, 4540 (1991)

${ }^{2}$ P. C. Hemmer and G. Stell, Phys. Rev. Lett. 24, 1284 (1970).

${ }^{3}$ A. Scala, M. R. Sadr-Lahijany, N. Giovambattista, S. V. Buldyrev, and H. E. Stanley, J. Stat. Phys. 100, 97 (2000).

${ }^{4}$ S. V. Buldyrev, G. Franzese, N. Giovambattista, G. Malescio, M. R. SadrLahijany, A. Scala, A. Skibinsky, and H. E. Stanley, Physica A 304, 23 (2002)

${ }^{5}$ P. Camp, Phys. Rev. E 68, 061506 (2003).

${ }^{6}$ P. Camp, Phys. Rev. E 71, 031507 (2005).

${ }^{7}$ C. Buzano and M. Pretti, J. Chem. Phys. 119, 3791 (2003).

${ }^{8}$ A. Skibinsky, S. V. Buldyrev, G. Franzese, G. Malescio, and H. E. Stanley, Phys. Rev. E 69, 061206 (2005).

${ }^{9}$ G. Franzese, G. Malescio, A. Skibinsky, S. V. Buldyrev, and H. E. Stanley, Phys. Rev. E 66, 051206 (2002).

${ }^{10}$ A. Balladares and M. C. Barbosa, J. Phys.: Condens. Matter 16, 8811 (2004).

${ }^{11}$ A. B. de Oliveira and M. C. Barbosa, J. Phys.: Condens. Matter 17, 399 (2005).

${ }^{12}$ V. B. Henriques and M. C. Barbosa, Phys. Rev. E 71, 031504 (2005).

${ }^{13}$ V. B. Henriques, N. Guissoni, M. A. Barbosa, M. Thielo, and M. C. Barbosa, Mol. Phys. 103, 3001 (2005).

${ }^{14}$ E. A. Jagla, "Phase behavior of a system of particles with core collapse," Phys. Rev. E 58, 1478 (1998).

${ }^{15}$ N. B. Wilding and J. E. Magee, "Phase behavior and thermodynamic anomalies of core-softened fluids," Phys. Rev. E 66, 031509 (2002).

${ }^{16}$ S. Maruyama, K. Wakabayashi, and M. A. Oguni, Aip Conf. Proc. 708, 675 (2004).

${ }^{17}$ R. Kurita and H. Tanaka, Science 206, 845 (2004).

${ }^{18}$ L. Xu, P. Kumar, S. V. Buldyrev, S.-H. Chen, P. Poole, F. Sciortino, and H. E. Stanley, Proc. Natl. Acad. Sci. U.S.A. 102, 16558 (2005).
${ }^{19}$ A. B. de Oliveira, P. A. Netz, T. Colla, and M. C. Barbosa, J. Chem. Phys. 124, 084505 (2006).

${ }^{20}$ A. B. de Oliveira, P. A. Netz, T. Colla, and M. C. Barbosa, J. Chem. Phys. 125, 124503 (2006).

${ }^{21}$ A. B. de Oliveira, M. C. Barbosa, and P. A. Netz, Physica A 386, 744 (2007).

${ }^{22}$ A. B. de Oliveira, P. A. Netz, and M. C. Barbosa, Eur. Phys. J. B 64, 481 (2008).

${ }^{23}$ A. B. de Oliveira, G. Franzese, P. A. Netz, and M. C. Barbosa, J. Chem. Phys. 128, 064901 (2008).

${ }^{24}$ A. B. de Oliveira, P. A. Netz, and M. C. Barbosa, Europhys. Lett. 85, 36001 (2009).

${ }^{25}$ N. V. Gribova, Y. D. Fomin, D. Frenkel, and V. N. Ryzhov, Phys. Rev. E 79, 051202 (2009)

${ }^{26}$ E. Lomba, N. G. Almarza, C. Martin, and C. McBride, J. Chem. Phys. 126, 244510 (2007)

${ }^{27}$ D. Y. Fomin, N. V. Gribova, V. N. Ryzhov, S. M. Stishov, and D. Frenkel, J. Chem. Phys. 129, 064512 (2008)

${ }^{28}$ G. S. Kell, J. Chem. Eng. Data 20, 97 (1975).

${ }^{29}$ C. A. Angell, E. D. Finch, and P. Bach, J. Chem. Phys. 65, 3063 (1976).

${ }^{30}$ T. Tsuchiya, J. Phys. Soc. Jpn. 60, 227 (1991).

${ }^{31}$ C. A. Angell, R. D. Bressel, M. Hemmatti, E. J. Sare, and J. C. Tucker, Phys. Chem. Chem. Phys. 2, 1559 (2000).

${ }^{32}$ R. Waler, Essays of Natural Experiments (Johnson Reprint, New York, 1964).

${ }^{33}$ R. J. Speedy and C. A. Angell, J. Chem. Phys. 65, 851 (1976).

${ }^{34} \mathrm{H}$. Kanno and C. A. Angell, "Water- anomalous compressibilities to 1.9 kbar and correlation with supercooling limits," J. Chem. Phys. 70(9), 40084016 (1979).

${ }^{35}$ C. A. Angell, M. Oguni, and W. J. Sichina, J. Phys. Chem. 86, 998 (1982).

${ }^{36}$ E. Tombari, C. Ferrari, and G. Salvetti, "Heat capacity anomaly in a large sample of supercooled water," Chem. Phys. Lett. 300, 749-751 (1999).

${ }^{37} \mathrm{H}$. Thurn and J. Ruska, J. Non-Cryst. Solids 22, 331 (1976).

${ }^{38}$ CRC Handbook of Chemistry and Physics, 65th ed., edited by R. C. Weast (CRC Press, Boca Raton, Florida, 1984).

${ }^{39}$ G. E. Sauer and L. B. Borst, Science 158, 1567 (1967).

${ }^{40}$ S. J. Kennedy and J. C. Wheeler, J. Chem. Phys. 78, 1523 (1983).

${ }^{41}$ R. Sharma, S. N. Chakraborty, and C. Chakravarty, J. Chem. Phys. 125, 204501 (2006).

${ }^{42}$ M. S. Shell, P. G. Debenedetti, and A. Z. Panagiotopoulos, Phys. Rev. E 66, 011202 (2002).

${ }^{43}$ P. H. Poole, M. Hemmati, and C. A. Angell, Phys. Rev. Lett. 79, 2281 (1997).

${ }^{44}$ S. Sastry and C. A. Angell, Nature Mater. 2, 739 (2003).

${ }^{45}$ P. H. Poole, F. Sciortino, U. Essmann, and H. E. Stanley, Nature (London) 360, 324 (1992)

${ }^{46}$ O. Mishima and H. E. Stanley, Nature (London) 396, 329 (1998).

${ }^{47}$ G. Franzese, G. Malescio, A. Skibinsky, S. V. Buldyrev, and H. E. Stanley, Nature (London) 409, 692 (2001).

${ }^{48}$ P. Vilaseca and G. Franzese, J. Chem. Phys. 133, 084507 (2010).

${ }^{49}$ J. Y. Abraham, S. V. Buldyrev, and N. Giovambattista, J. Phys. Chem. B 115, 14229 (2011)

${ }^{50}$ G. Malescio, G. Franzese, A. Skibinsky, S. V. Buldyrev, and H. E. Stanley, Phys. Rev. E 71, 061504 (2005).

${ }^{51}$ Z. Y. Yan, S. V. Buldyrev, P. Kumar, N. Giovambattista, and H. E. Stanley, Phys. Rev. E 77, 042201 (2008).

${ }^{52}$ N. M. Barraz, Jr., E. Salcedo, and M. C. Barbosa, J. Chem. Phys. 135, 104507 (2011)

${ }^{53}$ J. da Silva, E. Salcedo, A. B. Oliveira, and M. C. Barbosa, J. Phys. Chem. 133, 244506 (2010).

${ }^{54}$ N. M. Barraz, Jr., E. Salcedo, and M. C. Barbosa, J. Chem. Phys. 131, 094504 (2009)

${ }^{55}$ E. Salcedo, A. B. de Oliveira, N. M. Barraz, Jr., C. Chakravarty, and M. C. Barbosa, J. Chem. Phys. 135, 044517 (2011).

${ }^{56}$ J. R. Errington and P. G. Debenedetti, "Relationship between structural order and the anomalies of liquid water," Nature (London) 409, 318 (2001).

${ }^{57}$ J. E. Errington, P. G. Debenedetti, and S. Torquato, J. Chem. Phys. 118, 2256 (2003). 